\title{
Asymptotic Stability for an Axis-Symmetric Ohmic Heating Model in Thermal Electricity
}

\author{
Anyin Xia, ${ }^{1}$ Mingshu Fan, ${ }^{2}$ and Shan $\mathrm{Li}^{3}$ \\ ${ }^{1}$ School of Mathematics and Computer Engineering, Xihua University, Chengdu 610039, China \\ ${ }^{2}$ Department of Mathematics, Jincheng College of Sichuan University, Chengdu 611731, China \\ ${ }^{3}$ Business School, Sichuan University, Chengdu 610064, China
}

Correspondence should be addressed to Shan Li; lishan@scu.edu.cn

Received 12 June 2013; Accepted 4 July 2013

Academic Editor: Alain Miranville

Copyright (C) 2013 Anyin Xia et al. This is an open access article distributed under the Creative Commons Attribution License, which permits unrestricted use, distribution, and reproduction in any medium, provided the original work is properly cited.

\begin{abstract}
The asymptotic behavior of the solution for the Dirichlet problem of the parabolic equation with nonlocal term $u_{t}=u_{r r}+u_{r} / r+$ $f(u) /\left(a+2 \pi b \int_{0}^{1} f(u) r d r\right)^{2}$, for $0<r<1, t>0, u(1, t)=u^{\prime}(0, t)=0$, for $t>0, u(r, 0)=u_{0}(r)$, for $0 \leq r \leq 1$. The model prescribes the dimensionless temperature when the electric current flows through two conductors, subject to a fixed potential difference. One of the electrical resistivity of the axis-symmetric conductor depends on the temperature and the other one remains constant. The main results show that the temperature remains uniformly bounded for the generally decreasing function $f(s)$, and the global solution of the problem converges asymptotically to the unique equilibrium.
\end{abstract}

\section{Introduction}

In this paper, we consider the asymptotic behavior for the following initial boundary value problem:

$$
\begin{gathered}
u_{t}=u_{r r}+\frac{u_{r}}{r}+\frac{f(u)}{\left(a+2 \pi b \int_{0}^{1} f(u) r d r\right)^{2}}, \\
\text { for } 0<r<1, \quad t>0, \\
u(1, t)=u^{\prime}(0, t)=0, \quad \text { for } t>0, \\
u(r, 0)=u_{0}(r), \quad \text { for } 0 \leq r \leq 1,
\end{gathered}
$$

where $a$ and $b$ are some positive parameters. $f$ is a continuous, positive, and decreasing function, and the initial data $u_{0}(r)$ is a decreasing smooth positive function.

The original motivation for studying such problems comes from the plasma Ohmic heating process. The plasma is an electrical axis-symmetric conductor and so it could be heated by passing a current through it. This is called Ohmic heating and it is the same kind of heating that occurs in thermistors. We consider that the axis-symmetric conductor $A$ is a part of simple circuit in series with another constant one, $B$ and a constant voltage $E$ is applied (see Figure 1). Let $u(x, t)$ and $\rho(u)$ be the temperature and the electrical resistivity of the conductor $A$, respectively.

Here, the conductor $A \subset \mathbb{R}^{3}$ is a prismatic one with the length $L$ and the cross-sectional area $S$, and the length of conductor $B$ is $L^{\prime}$. Assume that the diameter of the crosssection $D$ is much less than $L$ and the temperature $u(x, t)$ is independent of the variable $x_{1}$. Suppose that the curved surface of the conductor $A, \Gamma_{0}$ is well thermal, and we can specify

$$
u=0 \quad \text { on } \Gamma_{0} \text {. }
$$

Based on the derivation in [1] (see also [2, 3]), we get the temperature $u$ of the material which satisfies the following:

$$
\begin{gathered}
u_{t}-\Delta u=\frac{f(u)}{\left(a+b \int_{D} f(u) d x\right)^{2}}, \quad x \in D, t>0, \\
u(x, t)=0, \quad x \in \partial D, t>0, \\
u(x, 0)=u_{0}(x), \quad x \in D,
\end{gathered}
$$




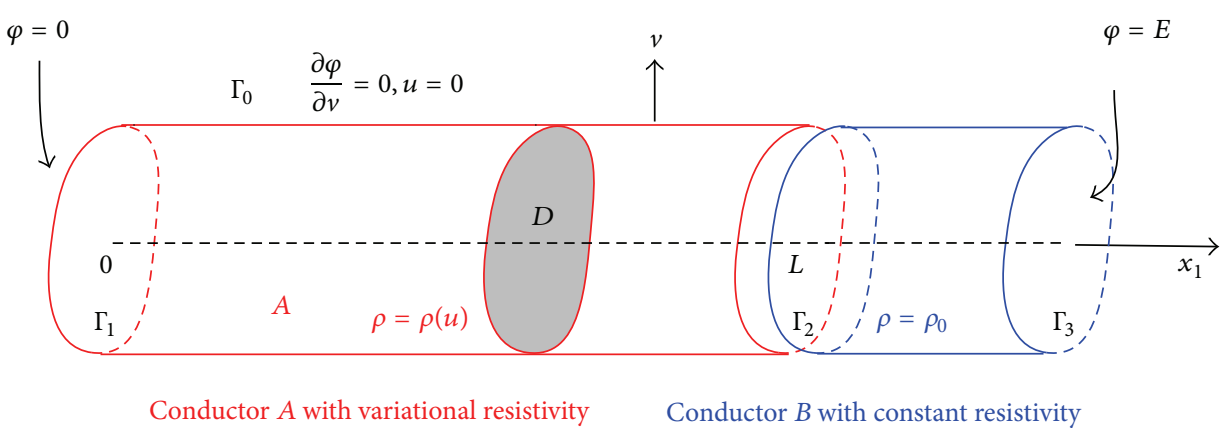

FIGURE 1: Electric current flows through two conductors.

where $f(u)=\rho^{-1}(u), a=L / E$, and $b=\left(\rho_{0} / E\right) L^{\prime}$. The initial data $u_{0}(x)$ is a positive, smooth function and satisfies $u_{0}(x)=0$ on $\partial D$.

In this paper, we focus on the problem (3) in radially symmetric case. So, we assume additionally that cross-section $D$ is a unit disk and the initial data be radially and decreasing, that is,

$$
u_{0}(x)=u_{0}(r), \quad u_{0}^{\prime}(r)<0,
$$

where $r=|x| \in(0,1)$. Thus, the problem in axis-symmetric case can be formulated into the problem (1). Furthermore, it is easy to see that the axis-symmetric solution to the problem (1) is radially decreasing (see [4]).

Here, we would like to address the works on the Ohmic heating model with one conductor $A$. The problem with only one conductor can be formulated into the following problem with different boundary conditions:

$$
u_{t}=\Delta u+\frac{\lambda f(u)}{\left(\int_{\Omega} f(u) d x\right)^{2}}, \quad \text { for } x \in \Omega, t>0 \text {, }
$$

where $\Omega \subset \mathbb{R}^{2}$ is an open, bounded domain, $f(u)$ is the electrical conductivity $\sigma(u)=1 / \rho(u)$, and the parameter $\lambda$ is a positive constant, which is depending upon the electric current or potential difference and also upon the "size" of the conductor (see $[1,5-9]$ ).

For problem (5), Lacey et al. have proved that if $f$ is an increasing function, then the blow up cannot take place (see $[1,10])$. If $f$ is a decreasing function, Lacey proved that comparison techniques was valid, by which he studied the asymptotic behavior of the solutions to (5) for special $f$ (see [1]). Taking the advantage of this fact, Lacey $[1,6]$ and Tzanetis [7] proved the occurrence of blow up for onedimensional model (5) and for the two-dimensional radially symmetric model (5), respectively. On the other hand, they proved that the global solution of (5) for some special $f(u)$, such as $f(u)=e^{-u}$, asymptotically converges to its unique steady state.

In [2], Du and Fan considered the nonlinear diffusion model for two conductors with one of the conductors remains constant. When $f$ is decreasing, they proved that comparison principle is valid and the solution of the model was always global in time. Furthermore, if $f$ is a decreasing exponential function, they proved that the solution of the problem converges asymptotically to the unique steady state. See also [3] for some results on asymptotic behavior of the global solution in one-dimensional case.

Inspired by these works, modified by the methods in [2], one can easily prove that the comparison principle for model (1) is valid and the solution of (1) is global in time. Finally, the main purpose of this paper is to give the asymptotic behavior and to show the asymptotic stability of the problem (1) with generally deceasing function $f(u)$.

Theorem 1. Assume that $f$ satisfies

$$
-s f^{\prime}(s)<f(s), \quad \text { for } s>0
$$

the solution $u(r, t)$ of the problem (1) converges asymptotically to the unique steady state $\omega(r)$, namely,

$$
u(r, t) \longrightarrow \omega(r), \quad \text { as } t \longrightarrow+\infty,
$$

for $0<r<1$.

Remark 2. The equations in models (1), (3), and (5) are semilinear parabolic equations with nonlocal sources. For the works on the global existence and blow up of nonlocal parabolic equations, the authors would like to refer to [11-14] and the references therein.

The analysis and techniques in this paper is based on the analysis for the ordinary differential equations and comparison arguments.

\section{The Asymptotic Stability for Problem (1)}

In this section, we will consider the asymptotic stability for the problem (1), and give the proof of Theorem 1 .

Proof of Theorem 1. Firstly, we deal with the local steady solution $\omega(r ; \mu)$ corresponding to the problem (1), consider

$$
\begin{gathered}
\omega^{\prime \prime}(r ; \mu)+\frac{1}{r} \omega^{\prime}(r ; \mu)+\mu f(\omega(r ; \mu))=0, \\
0<r<1, \\
\omega(1 ; \mu)=\omega^{\prime}(0 ; \mu)=0,
\end{gathered}
$$


with the positive parameter

$$
\mu=\frac{1}{\left(a+2 \pi b \int_{0}^{1} f(\omega(r ; \mu)) r d r\right)^{2}} .
$$

Moreover, multiplying $r$ on both sides of the equation in (8), and integrating over $[0,1]$ yield

$$
\int_{0}^{1} f(\omega(r ; \mu)) r d r=-\frac{\omega^{\prime}(1 ; \mu)}{\mu} .
$$

Multiplying $\omega^{\prime}(r ; \mu)$ on both sides of the equation in (8), and integrating over $[0,1]$ yields

$$
\begin{aligned}
\left(\omega^{\prime}(1 ; \mu)\right)^{2}= & 2 \mu \int_{0}^{M(\mu)} f(s) d s \\
& -2 \int_{0}^{1} \frac{\left(\omega^{\prime}(r ; \mu)\right)^{2}}{r} d r,
\end{aligned}
$$

where $M(\mu)=\omega(0 ; \mu)=\max _{r \in[0,1]} \omega(r ; \mu)$.

In view of $\omega^{\prime}(r ; \mu)<0$, for any $r \in(0,1)$, it follows from (11) that

$$
\omega^{\prime}(1 ; \mu)=-\sqrt{2 \mu \int_{0}^{M(\mu)} f(s) d s-2 \int_{0}^{1} \frac{\left(\omega^{\prime}(r ; \mu)\right)^{2}}{r} d r .}
$$

Combining this with (9) and (10) yields

$$
\begin{aligned}
\mu(a & \left.+2 \pi b \int_{0}^{1} f(\omega(r ; \mu)) r d r\right)^{2} \\
= & a^{2} \mu+\frac{4 \pi^{2} b^{2}\left(\omega^{\prime}(1 ; \mu)\right)^{2}}{\mu} \\
& -4 \pi a b \omega^{\prime}(1 ; \mu)=1 .
\end{aligned}
$$

It is easily seen that the problem (8) does not possess nontrivial solution with the parameter $\mu=0$. Namely, $\omega \equiv$ 0 and $M=0$ for $\mu=0$. Therefore, it follows from (11) that

$$
\lim _{\mu \rightarrow 0} \int_{0}^{1} \frac{\left(\omega^{\prime}(r ; \mu)\right)^{2}}{r} d r \longrightarrow 0,
$$

since $\left(\omega^{\prime}(1 ; \mu)\right)^{2}=2 \mu \int_{0}^{M(\mu)} f(s) d s-2 \int_{0}^{1}\left(\left(\omega^{\prime}(r ; \mu)\right)^{2} / r\right) d r>$ 0 and $\int_{0}^{1}\left(\left(\omega^{\prime}(r ; \mu)\right)^{2} / r\right) d r>0$.

Define the following:

$$
\begin{aligned}
F(\mu):= & a^{2} \mu+\frac{4 \pi^{2} b^{2}\left(\omega^{\prime}(1 ; \mu)\right)^{2}}{\mu} \\
& -4 \pi a b \omega^{\prime}(1 ; \mu)-1, \quad \text { for } \mu>0 .
\end{aligned}
$$

Then, it follows from a direct computation that $F(0+)<$ 0 and $F\left(1 / a^{2}\right)>0$. Then, there exists at least one root $\mu \in$ $\left(0,1 / a^{2}\right)$ to $(13)$
Making odd extension to the problem (8), we get

$$
\begin{gathered}
\omega^{\prime \prime}(r ; \mu)+\frac{1}{r} \omega^{\prime}(r ; \mu)+\mu f(\omega(r ; \mu))=0, \quad-1<r<1, \\
\omega( \pm 1 ; \mu)=0 .
\end{gathered}
$$

We claim that the differentiation of $\omega(r ; \mu)$ with respect to the parameter $\mu$ is always positive, namely, $\omega_{\mu}(r ; \mu)>0$, for any $0 \leq r \leq 1$. In fact, by differentiating on both sides of (16) respect to the parameter $\mu$, one gets

$$
\begin{aligned}
& -\omega_{\mu}^{\prime \prime}(r ; \mu)-\frac{1}{r} \omega_{\mu}^{\prime}(r ; \mu)-\mu f^{\prime}(\omega(r ; \mu)) \omega_{\mu}(r ; \mu) \\
& =f(\omega(r ; \mu))>0 .
\end{aligned}
$$

In view of $\omega_{\mu}( \pm 1 ; \mu)=0$, by comparison principle, we have $\omega_{\mu}(r ; \mu)>0$, for any $r \in[-1,1]$. Then, $M_{\mu}(\mu)=\omega_{\mu}(0$; $\mu)>0$.

Set

$$
\begin{aligned}
\varphi(\mu) & :=\frac{\left(\omega^{\prime}(1 ; \mu)\right)^{2}}{\mu} \\
& =\frac{2 \mu \int_{0}^{M(\mu)} f(s) d s-2 \int_{0}^{1}\left(\left(\omega^{\prime}(r ; \mu)\right)^{2} / r\right) d r}{\mu} .
\end{aligned}
$$

Let $z(r ; \mu)=\omega(r ; \mu) / \alpha, \alpha=\sqrt{\mu}$, we can rewrite

$$
\varphi(\mu)=\left(z^{\prime}(1 ; \mu)\right)^{2} .
$$

Then, it follows from $(8)$ that $z_{\alpha}(1 ; \mu)=z_{\alpha}^{\prime}(0 ; \mu)=z(1 ; \mu)=$ $z^{\prime}(0 ; \mu)$ and

$$
z^{\prime \prime}(r ; \mu)+\frac{z^{\prime}(r ; \mu)}{r}+\alpha f(\omega(r ; \mu))=0 .
$$

Differentiating on both sides of (20) with respect to the parameter $\alpha$, we have

$$
\begin{gathered}
z_{\alpha}^{\prime \prime}(r ; \mu)+\frac{z_{\alpha}^{\prime}(r ; \mu)}{r}+\alpha^{2} f^{\prime}(\omega(r ; \mu)) z_{\alpha}(r ; \mu) \\
=-f(\omega(r ; \mu))-f^{\prime}(\omega(r ; \mu)) \omega(r ; \mu) .
\end{gathered}
$$

If the condition (6) holds, then it follows from maximum principle and Hopf's boundary lemma that

$$
z_{\alpha}(r ; \mu)>0, \quad z_{\alpha}^{\prime}(1 ; \mu)<0 .
$$

Thus,

$$
\varphi^{\prime}(\mu)=\frac{1}{2 \alpha} \frac{d}{d \alpha}\left(z^{\prime}(1 ; \mu)\right)^{2}<0
$$

since $z^{\prime}(1 ; \mu)=\omega^{\prime}(1 ; \mu) / \alpha<0$. Therefore, we can conclude that $F^{\prime}(\mu)>0$, for any $\mu \in\left(0,1 / a^{2}\right)$. Furthermore, (13) possesses a unique root $\mu^{*}$ in $\left(0,1 / a^{2}\right)$, which shows that the problem possesses unique steady state $\omega\left(r, \mu^{*}\right)$. 
Next, we will show that the global solution of the problem (1) converges to its steady state. Inspired by the form of steady state $\omega\left(r ; \mu^{*}\right)$, we seek for a decreasing in time, upper solution of a form similar to the steady state $\bar{v}(r, t)=\omega(r, \bar{\mu}(t))=\bar{\omega}$, where $\bar{\mu}(t)$ will be determined later.

Then, one has

$$
\begin{aligned}
& \bar{v}_{t}-\bar{v}_{r r}-\frac{1}{r} \bar{v}_{r}-\frac{f(\bar{v})}{\left(a+2 \pi b \int_{0}^{1} r f(\bar{v}) d r\right)^{2}} \\
& =\bar{\omega}_{\bar{\mu}} \cdot \bar{\mu}^{\prime}(t)-\bar{\omega}_{r r}-\frac{1}{r} \bar{\omega}_{r} \\
& -\frac{f(\bar{\omega})}{\left(a+2 \pi b \int_{0}^{1} r f(\bar{\omega}) d r\right)^{2}} \\
& =\bar{\omega}_{\bar{\mu}} \cdot \bar{\mu}^{\prime}(t)-\frac{f(\bar{\omega})}{\left(a+2 \pi b \int_{0}^{1} r f(\bar{\omega}) d r\right)^{2}} \\
& \times\left[1-\bar{\mu}(t)\left(a+2 \pi b \int_{0}^{1} r f(\bar{\omega}) d r\right)^{2}\right] \\
& =\bar{\omega}_{\bar{\mu}}\left\{\bar{\mu}^{\prime}(t)-\frac{f(\bar{\omega})}{\bar{\omega}_{\bar{\mu}}\left(a+2 \pi b \int_{0}^{1} r f(\bar{\omega}) d r\right)^{2}}\right. \\
& \left.\times\left[1-\bar{\mu}(t)\left(a+2 \pi b \int_{0}^{1} r f(\bar{\omega}) d r\right)^{2}\right]\right\} .
\end{aligned}
$$

Since $u_{0}(r)$ and $u_{0}^{\prime}(r)$ are bounded in $[0,1]$, we can choose $\bar{\mu}(0)$, such that

$$
\bar{v}(r, 0)=\omega(r, \bar{\mu}(0)) \geq u_{0}(r) .
$$

Set

$$
\begin{aligned}
g(\bar{\mu}(t))= & \left(\inf _{r \in[0,1]} \frac{f(\bar{\omega})}{\bar{\omega}_{\bar{\mu}}}\right) \frac{1}{\left(a+2 \pi b \int_{0}^{1} r f(\bar{\omega}) d r\right)^{2}} \\
& \times\left[\bar{\mu}(t)\left(a+2 \pi b \int_{0}^{1} r f(\bar{\omega}) d r\right)^{2}-1\right] .
\end{aligned}
$$

Note that $g(\bar{\mu}(t)) \geq 0$, provided that $\bar{\mu}(t) \geq \mu^{*}$, where $\mu^{*}$ is the unique root of $(13)$, since $F(\mu)$ is increasing with respect to $\mu$. Thus, we can choose a decreasing function $\bar{\mu}(t)$ such that

$$
0 \geq \bar{\mu}^{\prime}(t) \geq-g(\bar{\mu}(t)), \quad \lim _{t \rightarrow \infty} \bar{\mu}(t)=\mu^{*}
$$

Then we have established an upper solution $\bar{v}(r, t)=\omega(r$; $\bar{\mu}(t))$ to the problem (1), which satisfies

$$
\bar{v}(r, t) \longrightarrow \omega\left(r ; \mu^{*}\right)=\omega(r), \quad \text { as } t \longrightarrow \infty .
$$

Similarly, we can construct a lower solution as $\underline{v}(r, t)=$ $\omega(r ; \mu(t))$, where $\mu(t)$ is increasing in time and tends to $\mu^{*}$. Finally, by the comparison principle, we obtain a pair of upper-lower solutions $(\bar{v}(r, t), \underline{v}(r, t))$, such that

$$
\underline{v}(r, t) \leq u(r, t) \leq \bar{v}(r, t), \quad \text { for } r \in[0,1], t>0,
$$

and it completes the proof of Theorem 1 .

\section{Acknowledgments}

This work is supported in part by NSFC Grant (11171236), SRFDP (no. 20100181120031), the Fundamental Research Funds for the Central Universities (0082604132187, skqy201224), and the fund of Key Disciplinary of Computer Software and Theory, Sichuan Grant (no. SZD0802-09-1).

\section{References}

[1] A. A. Lacey, "Thermal runaway in a non-local problem modelling Ohmic heating. I. Model derivation and some special cases," European Journal of Applied Mathematics, vol. 6, no. 2, pp. 127-144, 1995.

[2] L. Du and M. Fan, "Thermal runaway for a nonlinear diffusion model in thermal electricity," Discrete and Continuous Dynamical Systems A, vol. 33, no. 6, pp. 2349-2368, 2013.

[3] M. Fan and L. Du, "Asymptotic behavior for an Ohmic heating model in thermal electricity," Applied Mathematics and Computation, vol. 218, no. 22, pp. 10906-10913, 2012.

[4] B. Gidas, W. M. Ni, and L. Nirenberg, "Symmetry and related properties via the maximum principle," Communications in Mathematical Physics, vol. 68, no. 3, pp. 209-243, 1979.

[5] N. I. Kavallaris and T. Nadzieja, "On the blow-up of the non-local thermistor problem," Proceedings of the Edinburgh Mathematical Society. Series II, vol. 50, no. 2, pp. 389-409, 2007.

[6] A. A. Lacey, “Thermal runaway in a non-local problem modelling Ohmic heating. II. General proof of blow-up and asymptotics of runaway," European Journal of Applied Mathematics, vol. 6, no. 3, pp. 201-224, 1995.

[7] D. E. Tzanetis, "Blow-up of radially symmetric solutions of a non-local problem modelling Ohmic heating," Electronic Journal of Differential Equations, vol. 11, pp. 1-26, 2002.

[8] D. E. Tzanetis and P. M. Vlamos, "A nonlocal problem modelling ohmic heating with variable thermal conductivity," Nonlinear Analysis: Real World Applications, vol. 2, no. 4, pp. 443-454, 2001.

[9] D. E. Tzanetis and P. M. Vlamos, "Some interesting special cases of a non-local problem modelling Ohmic heating with variable thermal conductivity," Proceedings of the Edinburgh Mathematical Society. Series II, vol. 44, no. 3, pp. 585-595, 2001.

[10] J. W. Bebernes and A. A. Lacey, "Global existence and finite-time blow-up for a class of nonlocal parabolic problems," Advances in Differential Equations, vol. 2, no. 6, pp. 927-953, 1997.

[11] L. Du, "Blow-up for a degenerate reaction-diffusion system with nonlinear nonlocal sources," Journal of Computational and Applied Mathematics, vol. 202, no. 2, pp. 237-247, 2007.

[12] L. Du, C. Mu, and M. Fan, "Global existence and non-existence for a quasilinear degenerate parabolic system with non-local source," Dynamical Systems, vol. 20, no. 4, pp. 401-412, 2005. 
[13] L. Du and Z. Yao, "Localization of blow-up points for a nonlinear nonlocal porous medium equation," Communications on Pure and Applied Analysis, vol. 6, no. 1, pp. 183-190, 2007.

[14] M. Fan, C. Mu, and L. Du, "Uniform blow-up profiles for a nonlocal degenerate parabolic system," Applied Mathematical Sciences, vol. 1, no. 1-4, pp. 13-23, 2007. 


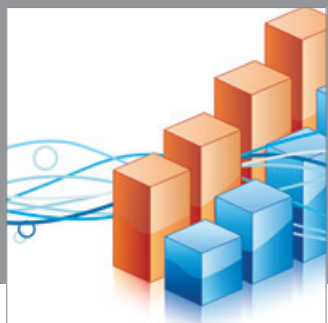

Advances in

Operations Research

mansans

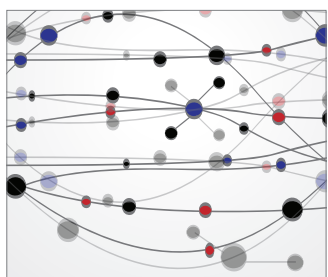

The Scientific World Journal
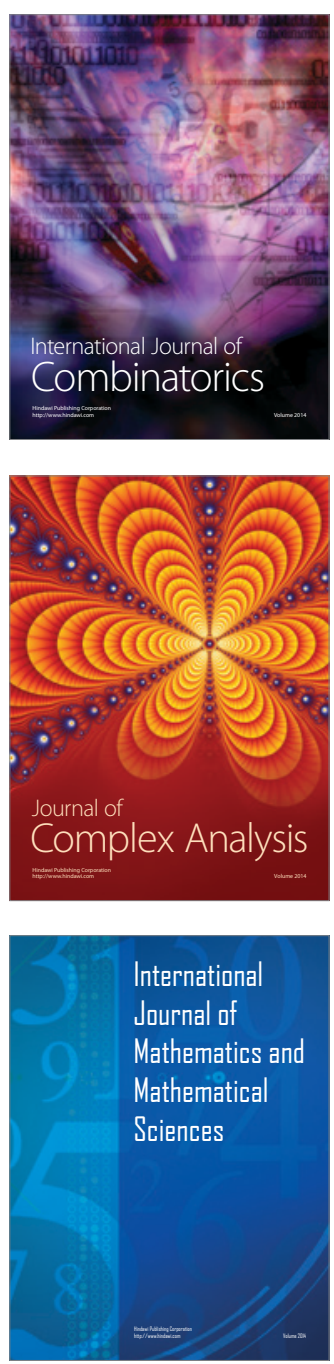
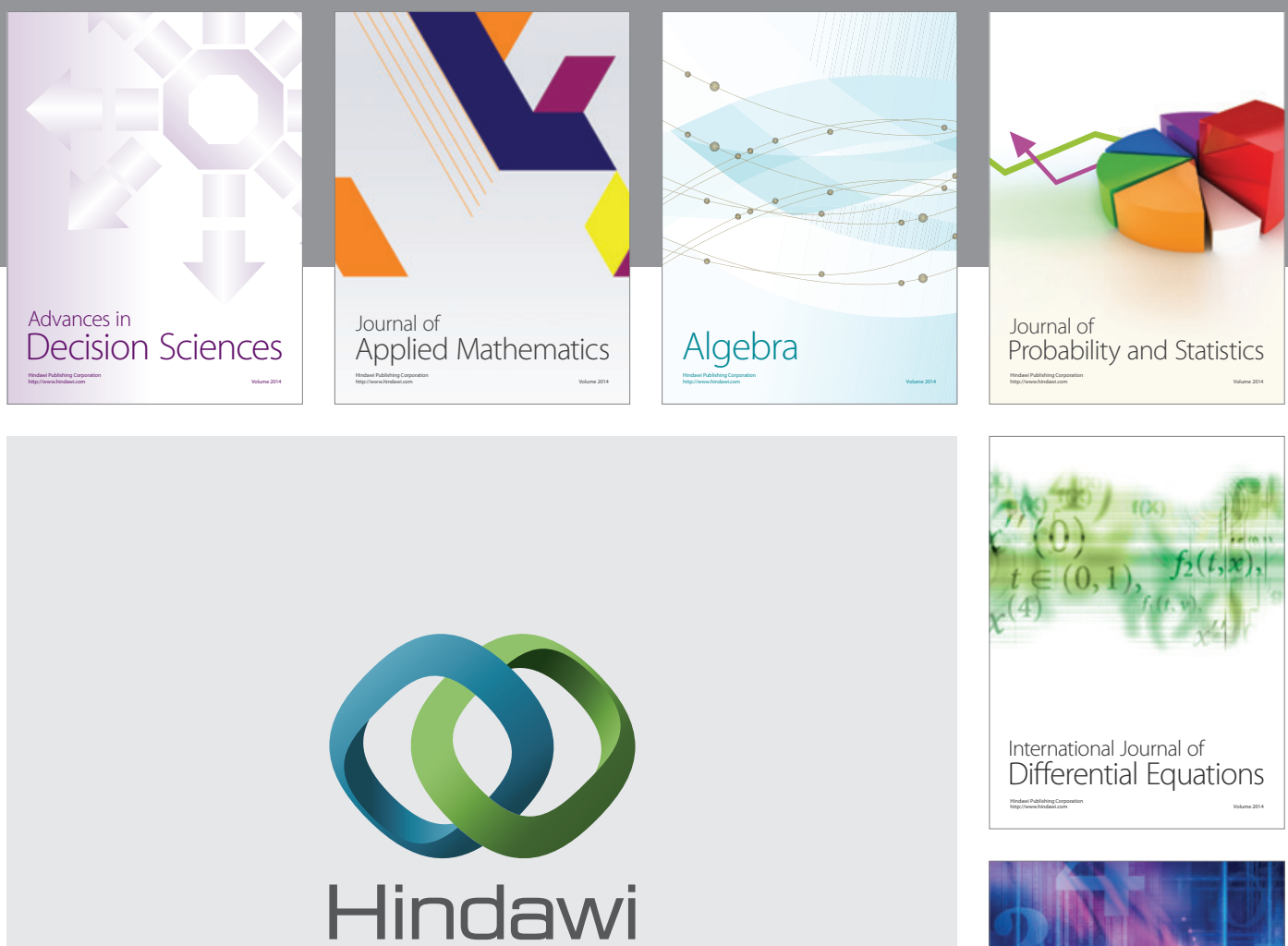

Submit your manuscripts at http://www.hindawi.com
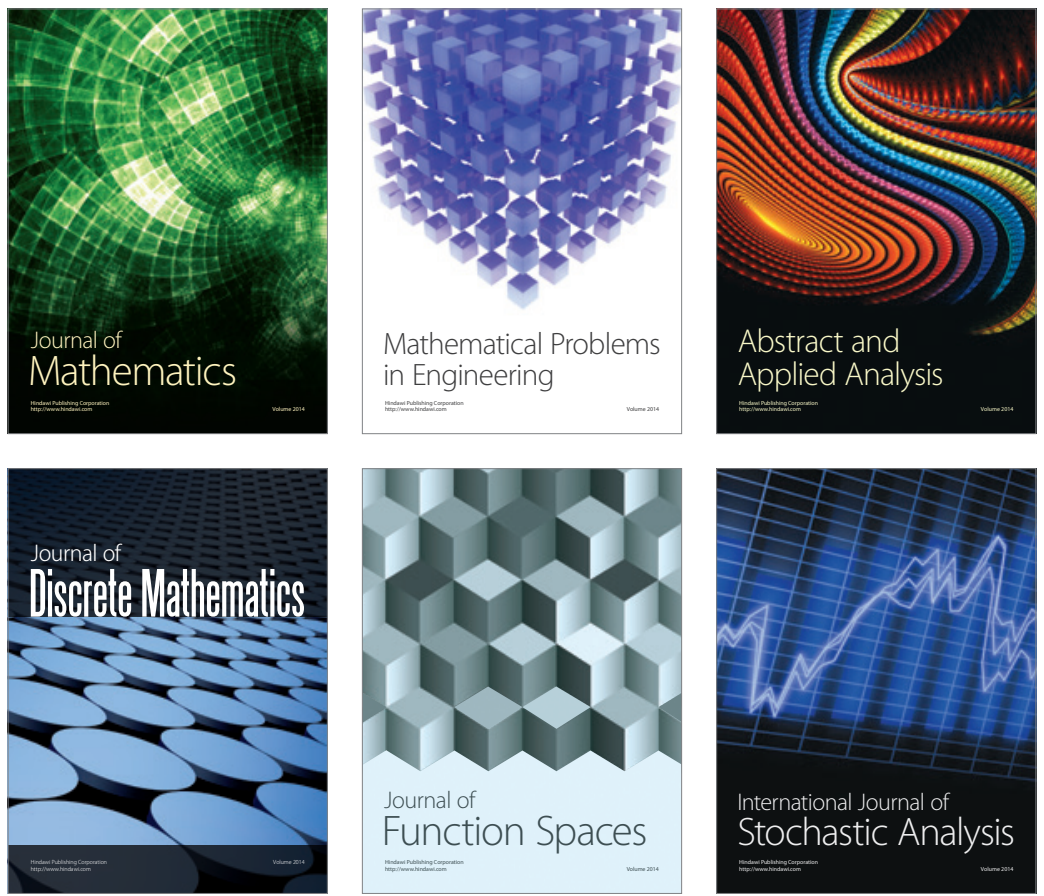

Journal of

Function Spaces

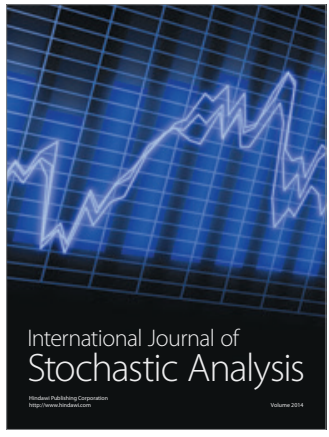

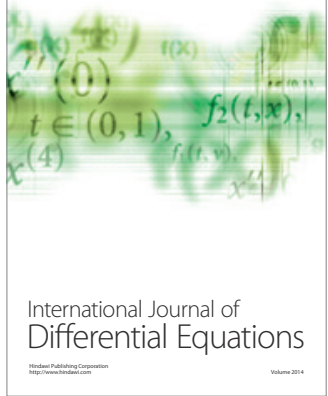
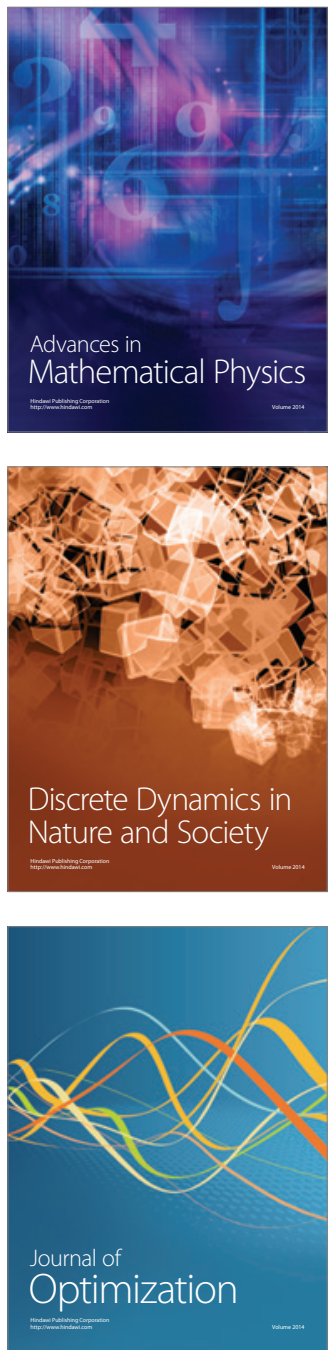